Tuberc Respir Dis 2012;72:486-492

Copyright(C)2012. The Korean Academy of Tuberculosis and Respiratory Diseases. All rights reserved.

\title{
Clinical Experience of Rigid Bronchoscopy in Single Center
}

\author{
Hyun Jin Kim, M.D. ${ }^{1}$, Sei Won Kim, M.D. ${ }^{1}$, Hye Yeon Lee, M.D. ${ }^{1}$, Hyeon Hui Kang, M.D. ${ }^{1}$, Ji Young Kang, \\ M.D. ${ }^{1}$, Ju Sang Kim, M.D. ${ }^{1}$, Myung Sook Kim, M.D. ${ }^{1}$, Seung Soo Kim, M.D. ${ }^{1}$, Jin Woo Kim, M.D. ${ }^{1}$, Hyeong \\ Gyu Yun, M.D. ${ }^{1}$, Chi Hong Kim, M.D. ${ }^{1}$, Kwan Hyoung Kim, M.D. ${ }^{1}$, Hwa Sik Moon, M.D. ${ }^{1}$, Kwang Jae Cho, \\ M.D. ${ }^{2}$, Seok Hwan Moon, M.D. ${ }^{3}$, Sang Haak Lee, M.D. ${ }^{1}$ \\ Departments of ${ }^{1}$ Internal Medicine, ${ }^{2}$ Otolaryngology-Head and Neck Surgery, and ${ }^{3}$ Thoracic and Cardiovascular Surgery, The \\ Catholic University of Korea School of Medicine, Seoul, Korea
}

\begin{abstract}
Background: The aim of this study was to analyze clinical situations requiring rigid bronchoscopy and evaluate usefulness of rigid bronchoscopic intervention in benign or malignant airway disorders.

Methods: We retrospectively reviewed 29 patients who underwent rigid bronchoscopy from November 2007 to February 2011 at St. Paul's Hospital, The Catholic University of Korea School of Medicine.

Results: Of the 29 patients, the most frequent underlying etiology was benign stenosis of trachea $(n=20)$. Of those 20 patients, 16 had post-intubation tracheal stenosis (PITS), 2 had tracheal stenosis due to inhalation burn (IBTS) and other 2 had obstructive fibrinous tracheal pseudomembrane (OFTP). Other etiologies were airway malignancy $(n=6)$, endobronchial stenosis due to tuberculosis $(n=2)$, and foreign body $(n=1)$. For treatment, silicone stent insertion was done in 16 cases of PITS and IBTS and mechanical removal was performed in 2 cases of OFTP. In 6 cases of malignant airway obstruction mechanical debulking was performed and silicone stents were inserted additionally in 2 cases. Balloon dilatation and electrocautery were used in 2 cases of endobronchial stenosis due to tuberculosis. In all cases of stent, airway obstructive symptom improved immediately. Granulation tissue formation was the most common complication.

Conclusion: Tracheal stenosis was most common indication and silicone stenting was most common procedure of rigid bronchoscopy in our center. Rigid bronchoscopic procedures, at least tracheal silicone stenting, should be included in pulmonary medicine fellowship programs because it is a very effective and indispensable method to relieve critical airway obstruction which needs training to learn.
\end{abstract}

Key Words: Bronchoscopy; Pulmonary Medicine; Tracheal Stenosis; Airway Obstruction

\section{Introduction}

Since Killian ${ }^{1}$ initially removed a pork bone within the right main bronchus by using esophagoscopy, the rigid bronchoscopy has more than a century of history. In the late 1960's, the rigid bronchoscopy had been declined due to discomfort and complications caused to

Address for correspondence: Sang Haak Lee, M.D.

Division of Pulmonology, Critical Care, and Sleep Medicine, Department of Internal Medicine, St. Paul's Hospital, The Catholic University of Korea, Jeonnong 1-dong, Dongdaemun-gu, Seoul 130-709, Korea

Phone: 82-2-961-4500, Fax: 82-2-968-7250

E-mail: mdlee@catholic.ac.kr

Received: Feb. 15, 2012

Revised: Mar. 26, 2012

Accepted: Apr. 26, 2012 patients after a flexible bronchoscope was introduced. However, the rigid bronchoscopy is steadily increasing again due to advanced bronchoscopic treatments, increasing number of lung cancer patients and advanced anesthesia and ventilation control from the mid-1980's ${ }^{2}$.

The rigid bronchoscopy is greatly effective and essential treatment in alleviating central airway obstruction. However, it is an invasive method conducted under general anesthesia requiring experienced doctors, and the method is not commonly performed in many hospitals. The authors of this paper aim to convey clinical experiences of rigid bronchoscopy conducted in a single institution. Base on this, the study examines indications and clinical usefulness of the treatment where the rigid bronchoscopy could be applied. 


\section{Materials and Methods}

\section{Subjects}

The study subjects were 29 patients underwent the rigid bronchoscopy from November 2007 to February 2011 in St. Paul's Hospital, The Catholic University of Korea. With 7〜47 month follow-up period, retrospective review was performed on clinical symptoms and treatment methods. St. Paul's Hospital has started the rigid bronchoscopy since November 2007, and this study passed deliberation of the Clinical Ethics Committee of St. Paul's Hospital (PIRB-00094_3-006).

\section{Surgical procedures of rigid bronchoscopy}

Clinical manifestation, simple chest X-ray, chest-computed tomography (CT), and a flexible bronchoscope were used for examination of airway lesions. All of the patients received rigid bronchoscopy under general anesthesia induced with injection of propofol. The intervention was performed by a single doctor. After inserting a rigid bronchoscope (Hopkins; Karl Storz, Tuttlingen, Germany), the lesion was examined by a flexible bronchoscope (EVIS BF 1T240 \& 1T260; Olympus, Tokyo, Japan). Depending on the type of lesions, electrocautery (electrosurgical unit [PSD-20], coagulation electrode [CD-6C-1], electrosurgical snare [SD-7C-1], electrosurgical knife [KD-31C-1]; Olympus), balloon catheter $(8 \sim 15 \mathrm{~mm}$ controlled radial expansion balloon; Boston Scientific, Natick, MA, USA), and other surgical devices were used to remove or dilate the lesions. Silicone stents were inserted in case needed. After the intervention, the site was examined using a flexible bronchoscope.

\section{Clinical observation after performing interventional bronchoscopy}

By examining clinical manifestation of a patient, simple chest X-ray, pulmonary function test, and a flexible bronchoscope, treatment effects and progression were verified. After the intervention, follow-up of each patient by flexible bronchoscopy was scheduled individually. Through flexible bronchoscopy, changes on in- terventional site, migration of stents, formation of granulation tissue, recurrent stenosis were examined. In addition, patients were treated through rigid or flexible bronchoscopy in case needed. In some cases, additional approaches were used in treatment such as a surgery.

\section{Statistical analysis}

Pulmonary function test of subjects before and after the insertion of silicone stents were compared by applying paired t-test. For statistical analysis, SPSS version 17.0 (SPSS Inc., Chicago, IL, USA) program was used and statistically significant difference was thought to be present when the p-value is less than 0.05 .

\section{Results}

\section{Characteristics of subjects}

The study subjects were 29 composed of 18 males and 11 females. The median age was 59 (age range, $20 \sim 78$ years). The most common causal diseases for performing rigid bronchoscopy were tracheal stenosis after endotracheal intubation, obstructive fibrinous tracheal pseudomembrane, and benign tracheal stenosis secondary to burn inhalation injury $(n=20)$. Other causal diseases were airway obstruction by malignant tumor $(n=6)$, tracheal stenosis due to tuberculosis and foreign body within the trachea (Table 1 ).

Table 1. Patient baseline characteristics

\begin{tabular}{lc}
\hline \multicolumn{1}{c}{ Characteristics } & No. $(\mathrm{n}=29)$ \\
\hline Age, median (range), yr & $59(20 \sim 78)$ \\
Gender & $\mathrm{M}: \mathrm{F}=18: 11$ \\
Etiology & \\
Benign tracheal stenosis & 20 \\
$\quad$ Post-intubation tracheal stenosis & 16 \\
$\quad$ Obstructive fibrinous tracheal pseudomembrane & 2 \\
Tracheal stenosis due to inhalation burn & 2 \\
Malignancy & 6 \\
Endobronchial stenosis due to tuberculosis & 2 \\
Foreign body & 1 \\
\hline
\end{tabular}

M: male; F: female. 


\section{Treatment by rigid bronchoscopy}

Among 18 cases of tracheal stenosis caused by endotracheal intubation and inhalation burn, silicone stents were inserted in 16 cases and T-tube insertion was performed in a case for treatment (Figures 1,2). In a case, insertion of a rigid bronchoscope was impossible since hyperextension of the neck was not secured in anatomical position. Among 16 cases of tracheal stenosis caused by endotracheal intubation, 8 cases underwent tracheostomy including T-tube before stent insertion. In a case, tracheal end-to-end anastomosis was performed but tracheal stenosis reoccurred. In the rest of 7 other cases, silicone stent insertion was implemented as an initial treatment. In two cases of obstructive fibrinous tracheal pseudomembrane, tracheal stenosis was alleviated by completely removing pseudomembrane using a rigid bronchoscope and a forcep. In 6 cases of malignant tumor, tumors were removed by performing snare and mechanical polypectomy using electrocautry through a rigid bronchoscope. In two cases, additional silicone stents were inserted on tracheal stenosis lesions after the
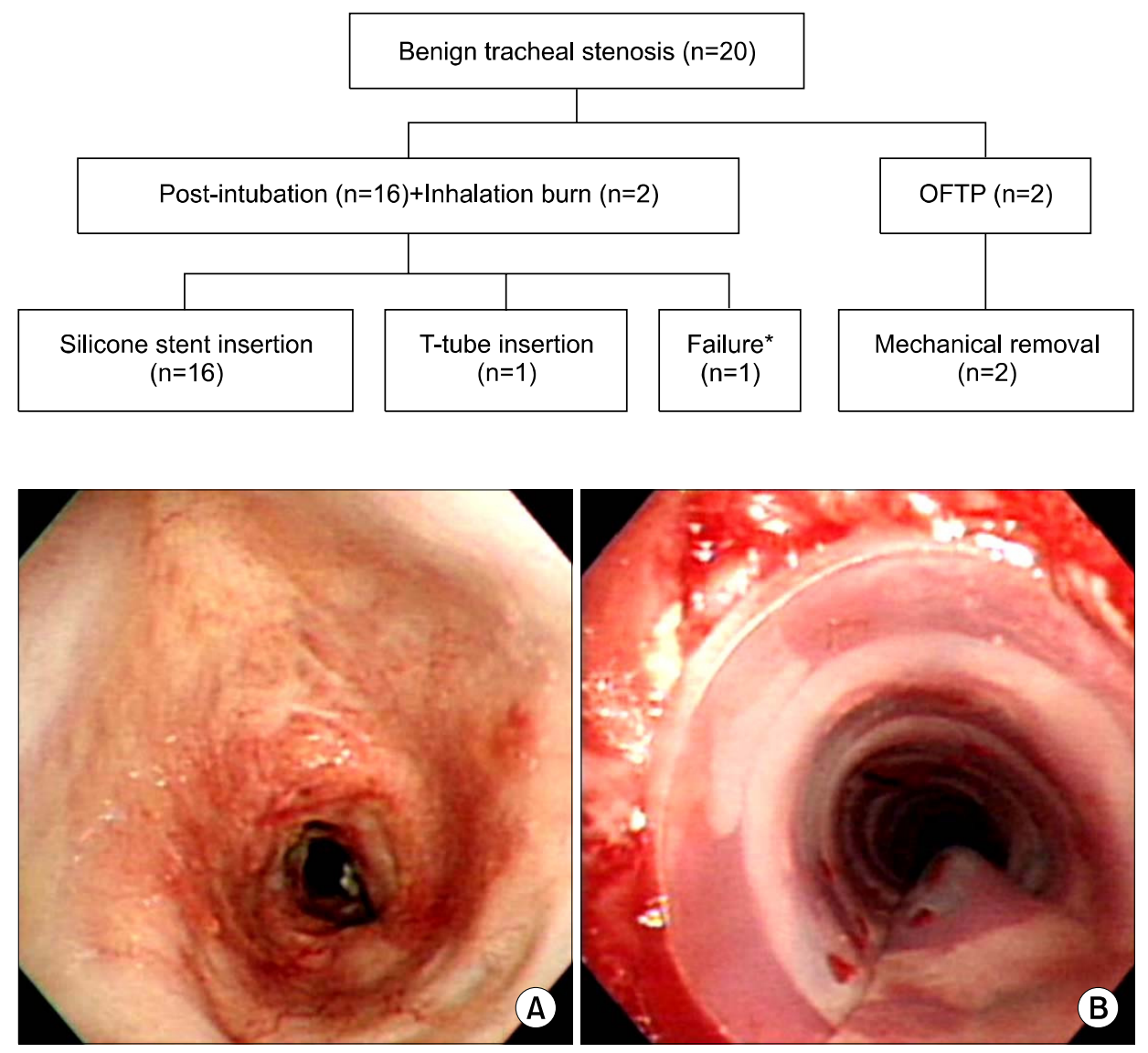

Figure 1. Rigid bronchoscopic intervention for benign tracheal stenosis. ${ }^{*}$ nsertion failure of rigid bronchoscopy due to anatomical problem (inability to withstand hyperextension of neck). OFTP: obstructive fibrinous tracheal pseudomembrane.

Figure 2. Representative bronchoscopic findings in a patient with post-intubation tracheal stenosis. (A) Baseline bronchoscopy showed marked tracheal stenosis. (B) After silicone stent insertion, bronchoscopy showed good patency in trachea.

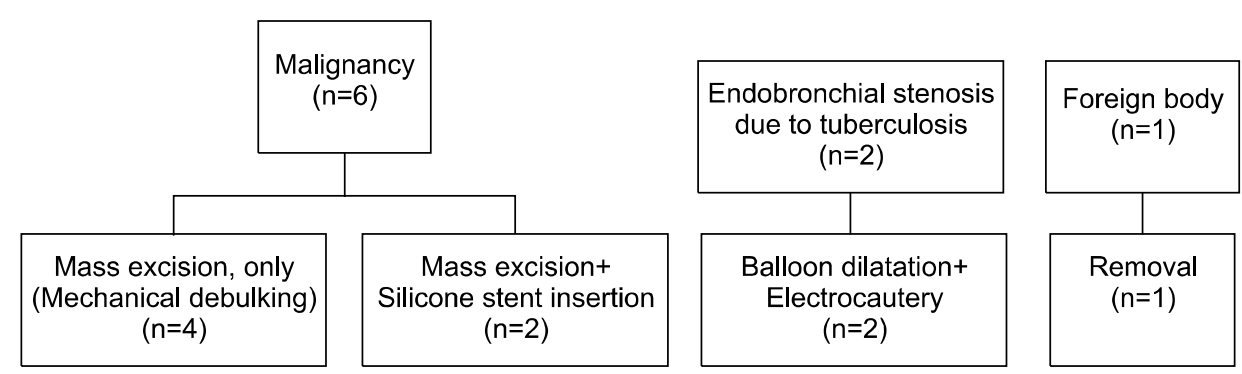

Figure 3. Rigid bronchoscopic intervention for other etiologies. 
removal of tumors. Moreover, in the other two cases of tracheal stenosis due to tuberculosis, balloon dilation and electrocautry was performed. Removal was conducted in a case of foreign body within the trachea (Figure 3).

\section{Clinical result after being treated with rigid bron- choscopy}

The average follow-up period was 27 months in the case of tracheal stenosis resulted from endotracheal intubation (range, 11 41 months); 23 months in inhalation burn cases (range, 22 24 months); 34 months in obstructive fibrinous tracheal pseudomembrane cases (range, 21 $\sim 47$ months); 15 months in the case of tracheal stenosis caused by malignant tumors (range, $7 \sim$ 30 months); 34 months in tracheal stenosis due to tuberculosis (range, 22 46 months); and 28 months in the case of foreign body within the trachea.

Sixteen cases of tracheal stenosis caused by endotracheal intubation and inhalation burn were treated by stent insertion. In 13 out of 16 cases, inserted silicone stents were maintained. In two cases of recurrent stenosis caused by repeated formation of granuloma and deteriorating breathing patterns, T-tube was inserted after eliminating stents. In one of the above cases, a patient was completely recovered from tracheal stenosis by conducting tracheal end-to-end anastomosis after eliminating stents when general physical condition was alleviated.

To confirm the clinical effectiveness among patients

Table 2. Change in pulmonary function after silicone stenting

\begin{tabular}{lccc}
\hline & $\begin{array}{c}\text { Baseline } \\
(\mathrm{n}=12)\end{array}$ & $\begin{array}{c}\text { After intervention } \\
(\mathrm{n}=12)\end{array}$ & $\mathrm{p}$-value* \\
\hline FVC, L & $3.24 \pm 1.04$ & $3.68 \pm 1.13$ & 0.005 \\
FVC, \% & $87.08 \pm 17.73$ & $104.25 \pm 29.20$ & 0.008 \\
FEV1, L & $1.85 \pm 0.79$ & $2.69 \pm 0.96$ & 0.009 \\
FEV1, \% & $67.33 \pm 22.31$ & $103.17 \pm 31.60$ & 0.013 \\
FEV1/FVC, \% & $58.00 \pm 19.08$ & $72.67 \pm 10.90$ & 0.011 \\
\hline
\end{tabular}

*Statistical significance test by paired t-test.

FVC: forced vital capacity; FEV1: forced expiratory volume in 1 second. who underwent silicone stents insertion, pulmonary function of 12 subjects were significantly improved when the pulmonary function test was performed on them before and after the intervention (Table 2). Pulmonary function test was performed less than one month and between 1 3 months after intervention for 4 patients each. In between 3 6 months and over a year period were 1 and 3 patients, respectively.

In all of the cases of tracheal stenosis resulted from malignant tumors, airway obstruction was immediately improved after the intervention through a rigid bronchoscope. Therefore, additional anti-cancer treatments were able to be performed after the elimination of tumors. In one of two cases where silicone stents were inserted after tumor resection, the lesion was alleviated after the additional radiotherapy was implemented. For that reason, stents were removed. In one of cases, radiotherapy was performed on squamous cell carcinoma of pulmonary parenchyma, the primary lesion, with maintaining stents. During follow-up period, two subjects died. In a case where a mass of lung cancer, which induced obstructive pneumonia, within the trachea was removed. The subject underwent anti-cancer treatments after pneumonia was relieved. However, the patient died due to the progress of lung carcinoma 23 months after the intervention. In the other case, tumors within the trachea and main bronchus were improved after the intervention. Although the radiotherapy was able to be carried on, the subject died due to advancement of the cancer 11 months after the intervention.

In two cases of tracheal stenosis induced by tuberculosis within the trachea, the lesion was relieved with just balloon dilation and electrocautery only. Clinical manifestations such as difficulty in breathing were immediately improved. In the case of foreign body within trachea, repeatedly occurring pneumonia was alleviated after the foreign body was removed.

\section{Complications after being treated with rigid bron- choscopy}

Regarding complications, 9 cases were the formation of granulation tissue related to stents; 3 cases were mi- 
Table 3. Complications after rigid bronchoscopic intervention

\begin{tabular}{|c|c|}
\hline & No. $(n=17)$ \\
\hline Granulation tissue formation & 9 \\
\hline \multirow{2}{*}{$\begin{array}{c}\text { For treatment, removal by biopsy forcep } \\
\text { changing to T-tube }\end{array}$} & 7 \\
\hline & 2 \\
\hline Stent migration & 3 \\
\hline Skin and soft tissue infection around traction site & 2 \\
\hline Mucostasis & 1 \\
\hline Airway heat burn & 1 \\
\hline Bleeding & 1 \\
\hline
\end{tabular}

Complications can be overlapped.

gration of stents; and 2 cases were infections at the stitched area for fixing stents. There was each case in mucous retention, tracheal burn injury, and severe bleeding, respectively (Table 3 ). In 3 cases of the stent migration, the position was adjusted. In the case of infected stitches, only one case was relieved simply with antibiotics only. In another case, the subject was treated with antibiotics and reinsertion of stents after removal of existing stents. In case of the mucous retention, diagnosis and mucus removal was performed through a flexible bronchoscope. In case of the severe bleeding occurred during tumor resection, the subject showed hypoxia temporarily. However, the patient stabilized by hemostasis after the removal of tumor mass.

\section{Discussion}

Bronchoscopy is a useful procedure in diagnosis and treatment of tracheal diseases. The indications of bronchoscopy for treatment purpose are commonly central airway obstruction in the trachea and main bronchus. Depending on the cause, tracheal diseases are classified into benign and malignant diseases. Specified causal diseases are tracheal stenosis after endotracheal intubation; benign tracheal stenosis due to complications of tuberculosis; foreign body within the trachea; and malignant tracheal stenosis induced by primary and metastatic tumors. Those are the major indications for bronchoscopy. Tracheal stenosis after endotracheal intubation, particularly accounted for the highest number of cases in this study, has been greatly declined after low pressure high volume endotracheal tube cuff was applied. However, it is still significant issue for a patient who needs long-term endotracheal intubation ${ }^{3}$.

Treatments performed through bronchoscopy are balloon dilation, laser therapy, electrocautery, cryotherapy, metal or silicone stents insertion and others. Although most of those treatments are implementable in flexible bronchoscopy, silicone stents insertion is only possible in rigid bronchoscopy under general anesthesia ${ }^{4,5}$. The advantages of silicone stent insertion are that repositioning and removal of stents are always possible. For those reasons, the therapy is particularly preferred on benign tracheal stenosis patients ${ }^{6-10}$. Although surgical operations are selectively performed for treatment of tracheal stenosis, silicone stent insertion within the trachea is the only treatment when operations cannot be conducted in case of unfavorable general physical condition and extensive scope of stenosis. Endoscopic treatment could be more useful in some cases where the condition of a patient is alleviated solely with endoscopic treatment $^{6-10}$. Although inconvenient procedure of general anesthesia is required in performing rigid bronchoscopy, the treatment method enables accurate intervention by securing steady view since coughing and movement of patients are not induced by trachea stimulation. Moreover, stable breathing is maintained by securing mechanical ventilation during intervention ${ }^{7,8}$.

According to clinical experience of the author in this paper, all of the patients who underwent silicone stent insertion through rigid bronchoscopy, exhibited immediate improvement in clinical manifestation. Significant improvement was shown in comparison between the results before and after the pulmonary function test. Consequently, retention, elimination of silicone stent, and other additional treatments were implementable according the condition of patients.

According to the experience of the authors, the most common complication induced by silicone stent insertion was granuloma around stent area and they were removable through flexible bronchoscopy in most of the cases. However, in case where recurrence of granuloma 
was severe, stents had to be removed in order to perform other interventions. In case where wheezing sound and breathing patterns were deteriorating, examination was essential with the use of a flexible bronchoscope $^{11}$. In other studies, the most common side effects after silicone stent insertion were migration of stent, formation of granuloma, obstruction of stents due to mucus, and similar tendencies were shown in this re$\operatorname{search}^{4,8-10}$.

According to the clinical experience in the cases of tracheal stenosis due to tumors, all of the patients were alleviated in terms of clinical manifestation by complete or partial resection of tumors through rigid bronchoscope. Consequently, additional chemotherapy and radiography were able to be carried on. Although, two out of six treated patients died during follow-up period, both of them were able to undergo anti-cancer treatment after rigid bronchoscopy intervention and the cause of death was the progression of cancer. Despite the result was acquired through observation on a minority of patients, the study is thought to be beneficial in enhancing the life quality and extending the life span by integrating the result with other research results ${ }^{12-15}$.

According to the clinical experience of the authors in two cases of tracheal stenosis due to tuberculosis, the lesion was relieved with just balloon dilation and electrocautery only. However, corresponding patients were thought to be possible because they had only web-like short interval tracheal stenosis. According to the studies made up to now, there are more cases where silicone stent insertion is require in treatments due to recurrent stenosis, bronchomalacia, etc. ${ }^{8,9}$.

Rigid bronchoscopy need to be performed under general anesthesia especially in case of patients with difficulty in breathing. Despite of its usefulness, many institutions do not perform the treatment in reality, since even pulmonology specialized doctors lack experience in conducting the procedure. However, treating lesions within the trachea using rigid bronchoscopy could be remarkably effective and much safer method compare to other methods. Therefore, the applicability is thought to be enhanced when rigid bronchoscopy for treatment purpose including silicone stent insertion is incorporated in residency training program of pulmonologists in respective institutions.

\section{References}

1. Killian G. Meeting of the Society of Physicians of Freiburg. Munch Med Wochenschr 1899;45:378.

2. Ayers ML, Beamis JF Jr. Rigid bronchoscopy in the twenty-first century. Clin Chest Med 2001;22:355-64.

3. Grillo HC, Cooper JD, Geffin B, Pontoppidan H. A low-pressure cuff for tracheostomy tubes to minimize tracheal injury: a comparative clinical trial. J Thorac Cardiovasc Surg 1971;62:898-907.

4. Fernando HC, Sherwood JT, Krimsky W. Endoscopic therapies and stents for benign airway disorders: where are we, and where are we heading? Ann Thorac Surg 2010;89:S2183-7.

5. Ranu H, Madden BP. Endobronchial stenting in the management of large airway pathology. Postgrad Med J 2009;85:682-7.

6. Lee P, Kupeli E, Mehta AC. Airway stents. Clin Chest Med 2010;31:141-50.

7. Casal RF. Update in airway stents. Curr Opin Pulm Med 2010;16:321-8.

8. Ryu YJ, Yu CM, Choi JC, Kwon YS, Kim H, Kim J, et al. Clinical experience of silicone airway stent in the management of benign tracheobronchial stenosis. Tuberc Respir Dis 2005;59:62-8.

9. Ryu YJ, Kim H, Yu CM, Choi JC, Kwon YS, Kwon OJ. Use of silicone stents for the management of post-tuberculosis tracheobronchial stenosis. Eur Respir J 2006; 28:1029-35.

10. Park HY, Kim H, Koh WJ, Suh GY, Chung MP, Kwon OJ. Natural stent in the management of post-intubation tracheal stenosis. Respirology 2009;14:583-8.

11. Chung FT, Lin SM, Chou CL, Chen HC, Liu CY, Yu CT, et al. Factors leading to obstructive granulation tissue formation after ultraflex stenting in benign tracheal narrowing. Thorac Cardiovasc Surg 2010;58:102-7.

12. Jeon K, Kim H, Yu CM, Koh WJ, Suh GY, Chung MP, et al. Rigid bronchoscopic intervention in patients with respiratory failure caused by malignant central airway obstruction. J Thorac Oncol 2006;1:319-23.

13. Furukawa K, Ishida J, Yamaguchi G, Usuda J, Tsutsui $\mathrm{H}$, Saito M, et al. The role of airway stent placement in the management of tracheobronchial stenosis caused by inoperable advanced lung cancer. Surg Today 2010;40:315-20. 
HJ Kim et al: A single center experience

14. Choi JC, Yu CM, Ryu YJ, Jeon K, Choi KA, Kwon OJ, et al. The role of endoscopic surgery for completely obstructive endobronchial benign tumor. Korean J Intern Med 2006;21:15-9.
15. Choi SH, Kang JY, Joo YB, Kim SK, Mo EY, Lee SH, et al. An endobronchial lipoma treated by bronchoscopic excision. Korean J Med 2011;80:337-42. 